\title{
A novel technique for measuring intraoperative bladder neck and urethral dimensions during robotic-assisted radical prostatectomy
}

\author{
Jo-Lynn Tan ${ }^{1}$; Thomas Whish-Wilson ${ }^{1,2}$; Nathan Papa ${ }^{3}$; Lih-Ming Wong 1,2 \\ ${ }^{1}$ St Vincent's Hospital, Department of Urology, Melbourne, Australia; ${ }^{2}$ University of Melbourne, Department of Surgery, \\ Melbourne, Australia; ${ }^{3}$ Monash University, School of Public Health and Preventive Medicine, Melbourne, Australia
}

Cite as: Tan J-L, Whish-Wilson T, Papa N, et al. A novel technique for measuring intraoperative bladder neck and urethral dimensions during robotic-assisted radical prostatectomy. Can Urol Assoc J 2021 February 12; Epub ahead of print. http://dx.doi.org/10.5489/cuaj.7067

Published online February 12, 2021

$* * *$

\section{Introduction}

Urinary incontinence (UI) remains an important cause of morbidity after radical prostatectomy (RP), bearing significant impact on quality of life regardless of oncologic and sexual functional outcomes. ${ }^{1}$

Urethral length (UL) has been found to predict continence recovery ${ }^{2-6}$ and where possible, many surgeons aim to maximise intraoperative urethral length during dissection without compromising oncological margins. ${ }^{7}$ However, due to study heterogeneity, current published data defining this parameter is limited.

In the current age of robotic surgery, it is now possible to measure anatomical variables in real-time and under high-image resolution. To our knowledge, no intraoperative anatomical technique or measurements are currently available to accurately guide or predict continence recovery after robotic-assisted RP (RARP). We describe a novel method of measuring intra-operative urethral and bladder neck $(\mathrm{BN})$ dimensions, and examine the impact of these dimensions on UI after RARP.

\section{Methods}

We retrospectively reviewed all RARP cases $(n=101)$ performed by a single surgeon between November 2015 to January 2019, with the aim of assessing practicality of intra-operative measurement of urethral dimensions. All consenting patients who underwent RARP with complete intraoperative video recordings, and 3- and 6-month post-op follow-up data were included. Baseline data included age at time of surgery, cardiorespiratory and metabolic comorbidities, PSA level, preop IPSS, post-operative Gleason score and pathological stage. Patient reported pad usage per day at 3 and 6 months after surgery was recorded. Full continence recovery was defined by usage of 1 or fewer pads a day.

To assess intra-operative BN diameter and urethral dimensions, a novel approach was employed. Each operation video was reviewed on two independent occasions by a surgical resident, with an average of two measurements taken for each parameter on each occasion. Still screenshots were taken from each video to obtain standardized operative views, defined as the time-point after en bloc resection of the prostate but prior to anastomosis formation, and after 1-3 sutures were placed 
on the posterior wall of the urethra to the $\mathrm{BN}$ (Figure 1a). This aimed to ensure that all measurements were consistently taken at a well-defined operative time point, and importantly, to minimize the effect of varying degrees of tissue traction on our measurements. For complete measurement standardization, all intraoperative images were taken when a $0^{\circ}$ laparoscopic camera lens was in use. The known width of an $18 \mathrm{~F}$ catheter $(6 \mathrm{~mm})$ was utilized as a measurement constant, ensuring image magnification was accounted for on all measurements. A digital ruler application (ScreenRuler v1.2) was used to perform the required measurements (Figure 1b).

UL was measured from the membranous urethra to the cut edge (Figure 1b). BN width and urethral width (UW) were similarly measured at this time point. UW was measured by taking the widest measurement between well-defined mucosal edges on either side of the urethra (Figure 1c).

Analysis of the relationship between UL and dichotomous continence measures were performed using Wilcoxon rank sum tests and multivariable logistic regression with bilateral nerve sparing (yes/no) entered as a covariate. To further explore the nature and shape of the relationship between intraoperative dimensions and probability of incontinence measures; UL, UW, and BN width were modeled as restricted cubic splines and the predicted probabilities graphed. Analysis was performed using Stata v 13.0 SE (College Station, TX) with statistical significance set at 0.05.

\section{Results}

After accounting for irretrievable videos due to damaged or incomplete files or loss to 3- or 6-month follow-up, 62 subjects were analysed.

Median age at surgery was 64.2 years. The median UL was $0.95 \mathrm{~cm}$ (IQR: $0.77-1.16)$, median BN width $1.24 \mathrm{~cm}$ (IQR: 0.99-1.79), and median urethral width $1.41 \mathrm{~cm}$ (IQR: 1.26-1.56). It was technically feasible to obtain all measurements of UL, UW and BN using the technique described.

At three months post-op, shorter UL was associated with use of $\geq 2$ pads/day (median $0.81 \mathrm{~cm}$ ) vs $\leq 1 \mathrm{pad} /$ day (median $1.04 \mathrm{~cm}$ ), $\mathrm{p}=0.008$. After adjustment for bilateral nerve-sparing, this relationship retained statistical significance $(\mathrm{p}=0.034)$. The predicted probability of needing $\geq 2$ pads/day at 3 and $\geq 1 \mathrm{pad} /$ day at 6 months generally decreased with increasing UL and UW while the corresponding predicted probabilities were stable across the domain of $\mathrm{BN}$ width values (Figure 2).

\section{Discussion}

Preservation of UL has been shown to improve continence outcomes. ${ }^{4-6}$ However, differences in methodology (MRI, USS) and timing of measurements (pre-, intra-, post-operative) hinders its practical definition and undermines its clinical utility.

In the current era of robotic surgery, surgeons have the advantage of operating with superior visual magnification, allowing for more precise intra-operative measurements to be performed. There is value in broadening the utility of current technologies to allow for intra-operative measurements of important anatomical variables such as urethral dimensions to optimize anatomical preservation. One study addressed the use of intra-operative urethral measurements via transrectal ultrasound to guide post-operative continence recovery, and highlighted that the preservation of distal continence mechanisms are important for continence recovery. ${ }^{4}$ However, this lacks precision compared to our novel method described. 
To our knowledge, our study is the first offering practical utility of real-time measurements during RARP. It is precise, quick and easy to perform. If a digital ruler application software is not available, measurements could be performed by passing a sterile ruler strip into the patient and using the same technique described in our study. Intraoperative measurements are a precise and clinically meaningful way of enabling surgeons, in real-time, to maximise preservation of UL thus optimizing patients' post-operative continence recovery.

Furthermore, our findings of UL as an important function of continence recovery after RARP add to the growing evidence that preservation of the UL is important to maximizing continence outcomes. ${ }^{3,5,7}$ Paparel et al (2009) measured UL using MRI and showed that a longer UL both before and after surgery, as well as preservation of UL during surgery correlated with earlier continence recovery. ${ }^{5}$ These findings were corroborated in larger studies including a meta-analysis, ${ }^{3,6}$ highlighting the importance of using meticulous dissection and surgical techniques for maximal UL preservation during RP, for achieving improved continence outcomes.

\section{Conclusions}

Our novel technique introduces a simple and reliable method of measuring intraoperative urethral and BN dimensions with greater precision. Furthermore, our findings support UL preservation for improving continence outcomes but further analysis with a larger series is required.

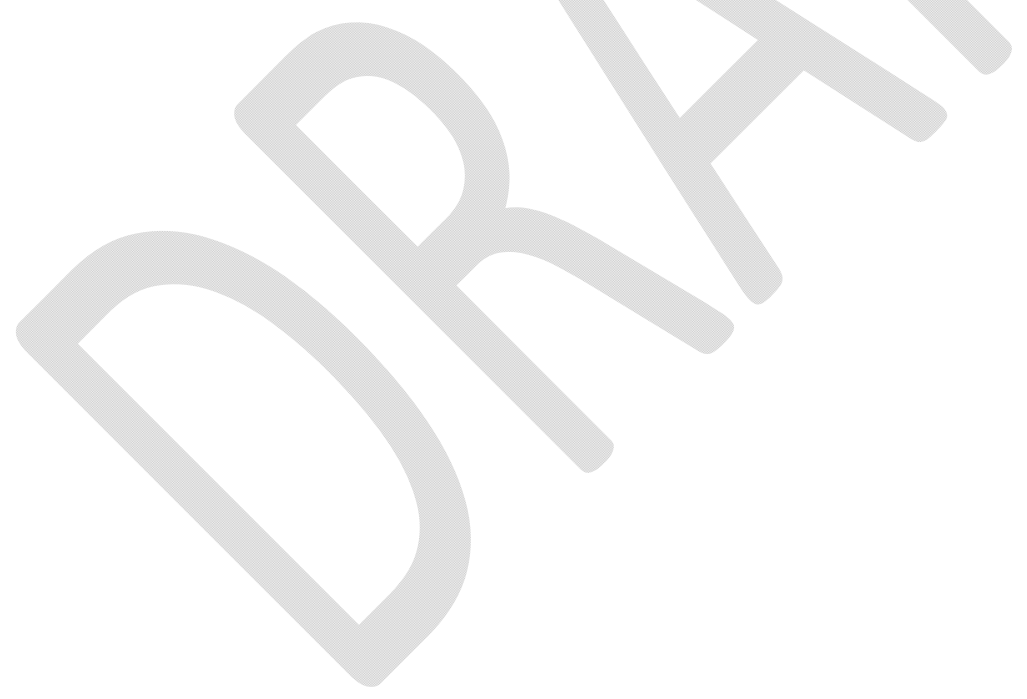




\section{References}

1. Xu AJ, Taksler GB, Llukani E, Lepor H. Long-Term Continence Outcomes in Men Undergoing Radical Prostatectomy: A Prospective 15-Year Longitudinal Study. J Urol. 2018;

2. Matsushita K, Kent MT, Vickers AJ, Von Bodman C, Bernstein M, Touijer KA, et al. Preoperative predictive model of recovery of urinary continence after radical prostatectomy. BJU Int. 2015;

3. Song W, Kim CK, Park BK, Jeon HG, Jeong BC, Seo S Il, et al. Impact of preoperative and postoperative membranous urethral length measured by 3 Tesla magnetic resonance imaging on urinary continence recovery after robotic-assisted radical prostatectomy. Can Urol Assoc J. 2017;

4. Hakimi AA, Faleck DM, Agalliu I, Rozenblit AM, Chernyak V, Ghavamian R. Preoperative and intraoperative measurements of urethral length as predictors of continence after robotassisted radical prostatectomy. J Endourol. 2011;

5. Paparel P, Akin O, Sandhu JS, Otero JR, Serio AM, Scardino PT, et al. Recovery of Urinary Continence after Radical Prostatectomy: Association with Urethral Length and Urethral Fibrosis Measured by Preoperative and Postoperative Endorectal Magnetic Resonance Imaging. Eur Urol. 2009;

6. Mungovan SF, Sandhu JS, Akin O, Smart NA, Graham PL, Patel MI. Preoperative Membranous Urethral Length Measurement and Continence Recovery Following Radical Prostatectomy: A Systematic Review and Meta-analysis. European Urology. 2017.

7. Schlomm T, Heinzer H, Steuber T, Salomon G, Engel O, Michl U, et al. Full functionallength urethral sphincter preservation during radical prostatectomy. Eur Urol. 2011;

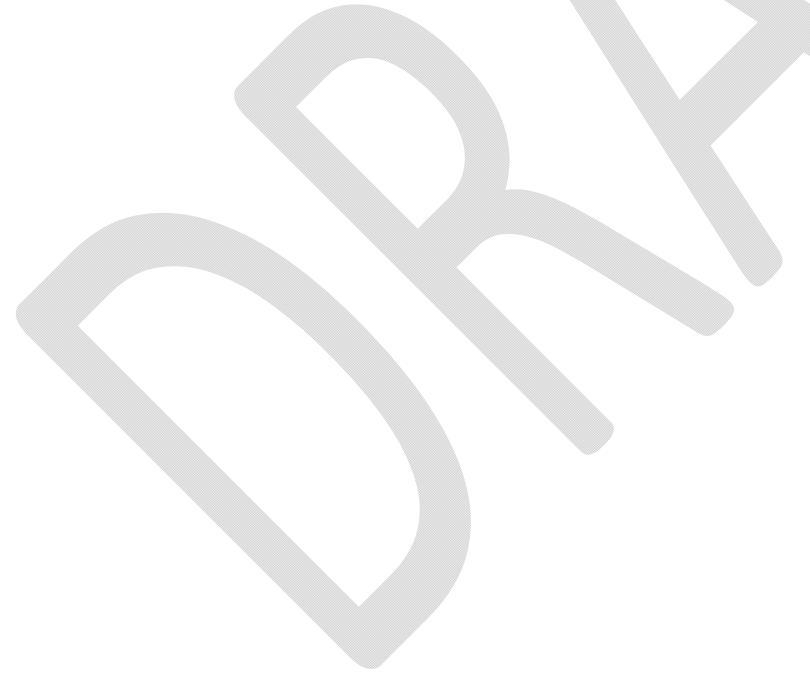




\section{Figures and Tables}

Fig. 1A. Standardized views capturing catheter width, bladder neck diameter, and urethral length.

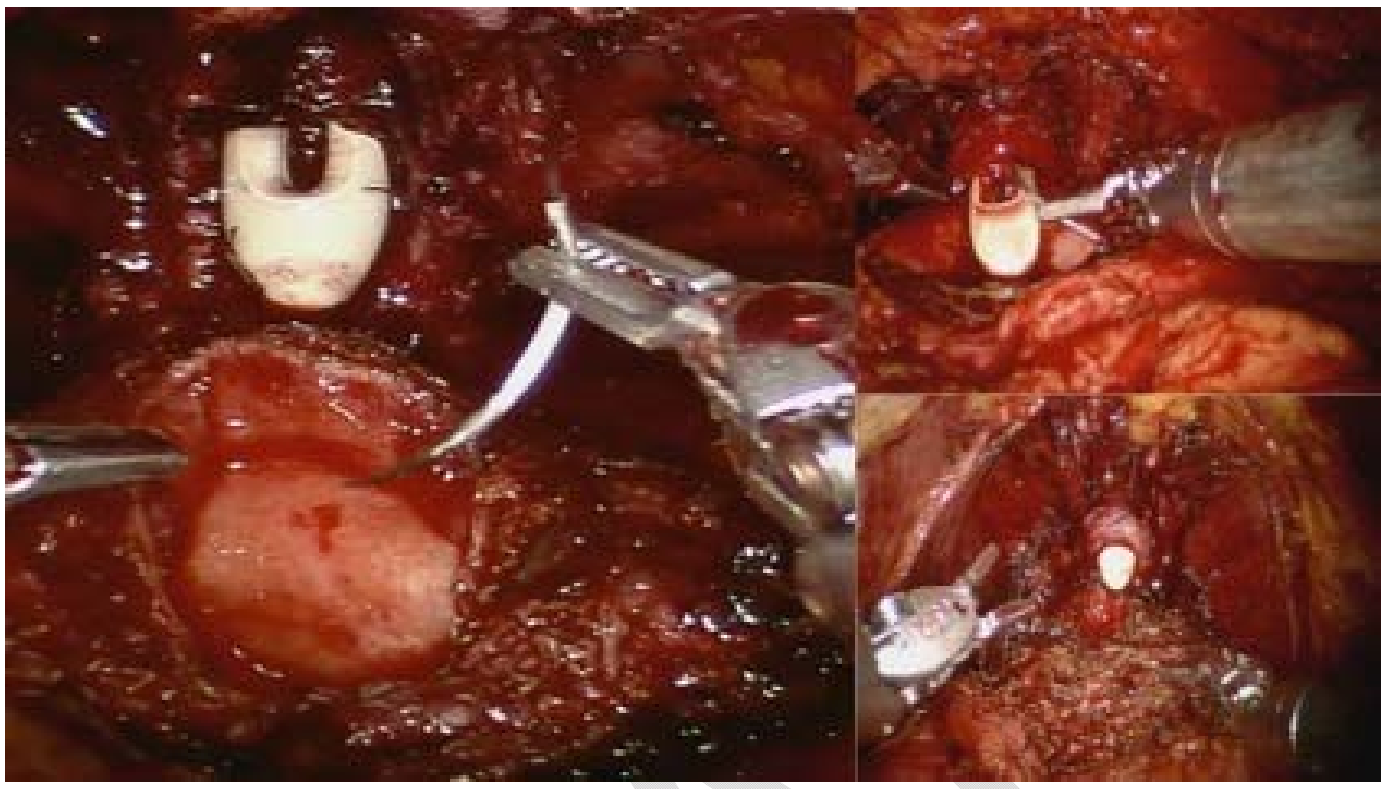

Fig. 1B. Measurement of urethral length and width using standardized intraoperative views.

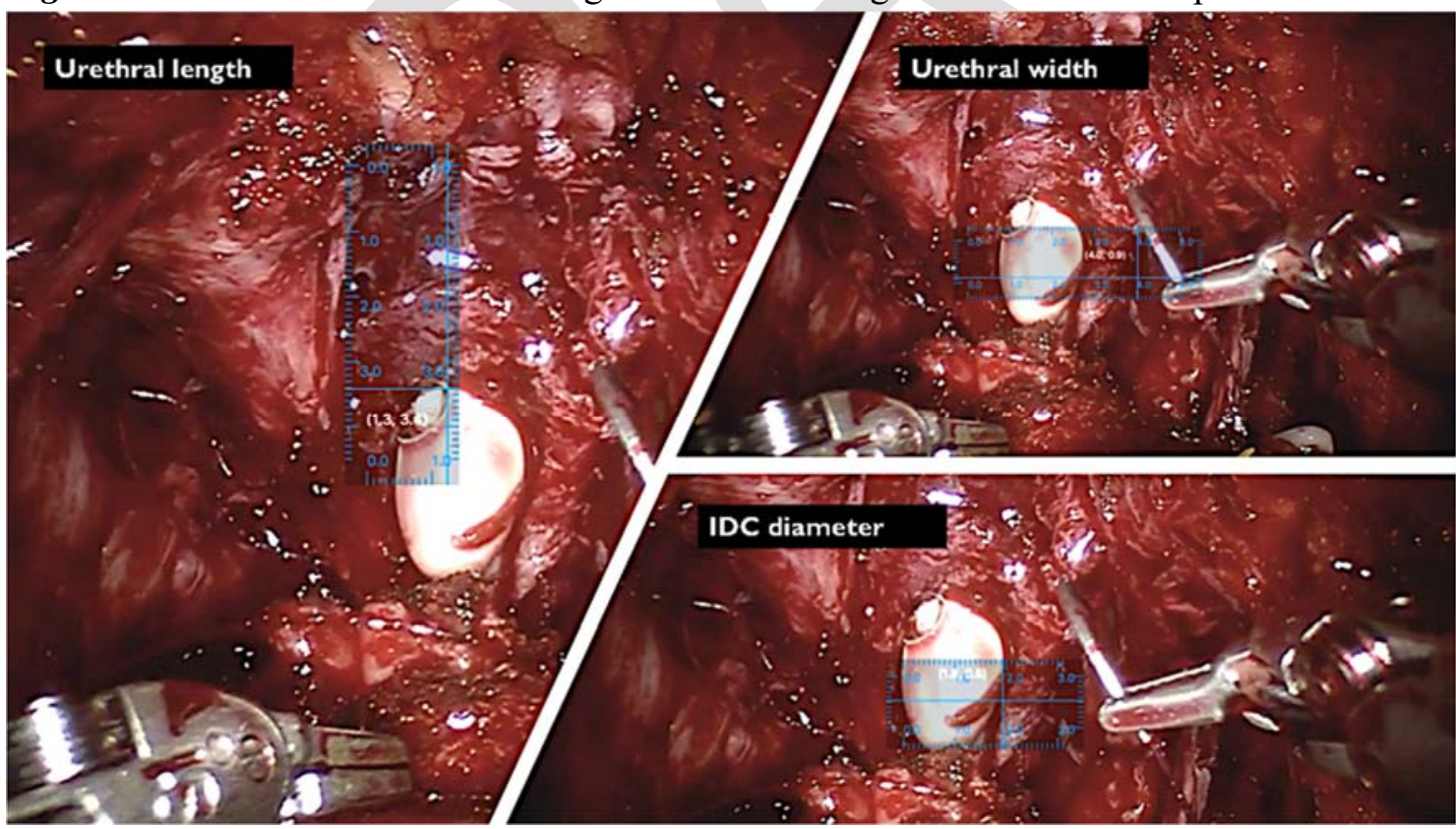


Fig. 1C. Measurement of bladder neck $(\mathrm{BN})$ width using digital ruler application.
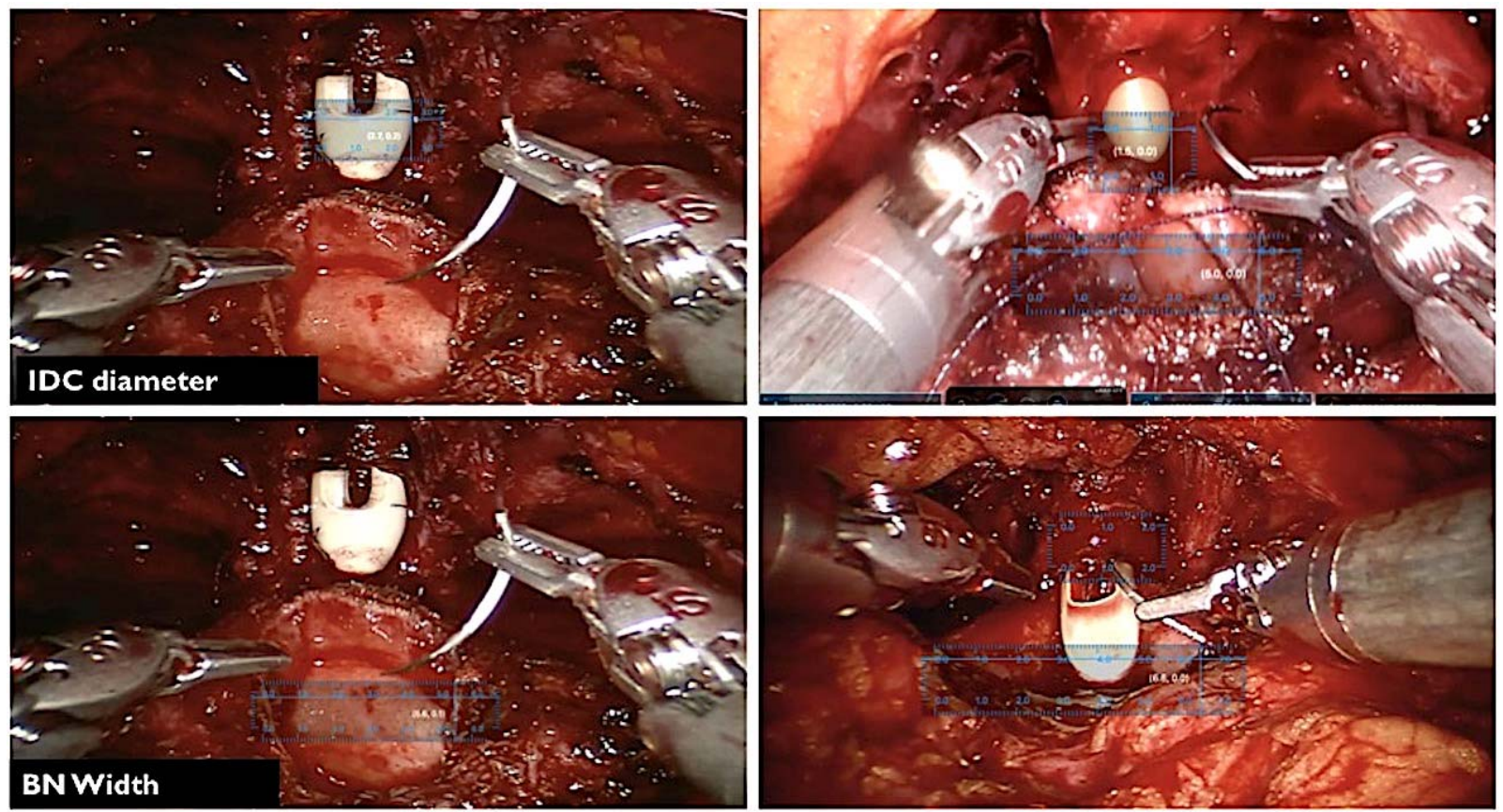

Fig. 2. (A) Predicted incontinence probabilities by urethral length; (B) predicted incontinence probabilities by urethral width; $(\boldsymbol{C})$ predicted incontinence probabilities by bladder neck width.
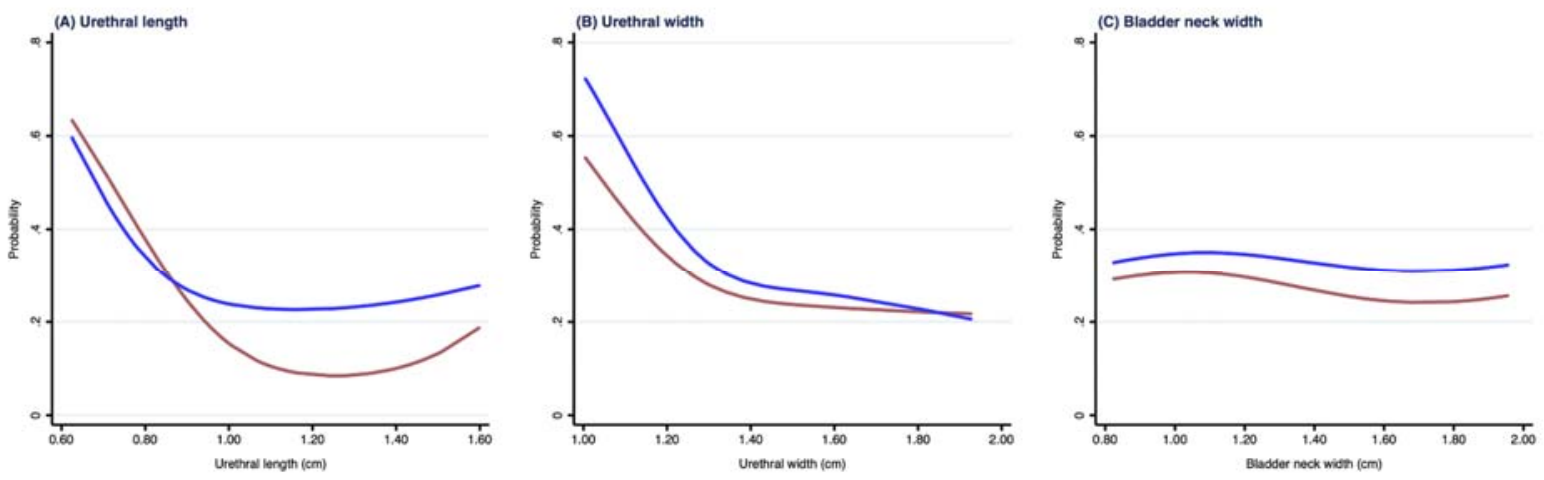

3 Months: 22 pads/day 6 Months: 21 pad/day 